\title{
Fast single-step fabrication of nanopores
}

This article has been downloaded from IOPscience. Please scroll down to see the full text article.

2009 Nanotechnology 20015302

(http://iopscience.iop.org/0957-4484/20/1/015302)

View the table of contents for this issue, or go to the journal homepage for more

Download details:

IP Address: 131.180.130.109

The article was downloaded on 08/08/2011 at 10:39

Please note that terms and conditions apply. 


\title{
Fast single-step fabrication of nanopores
}

\author{
P Chen, M-Y Wu, H W M Salemink and P F A Alkemade \\ Kavli Institute of Nanoscience, Delft University of Technology, Lorentzweg 1, 2628 CJ Delft, \\ The Netherlands \\ E-mail: P.Chen@tudelft.nl
}

Received 12 August 2008, in final form 23 October 2008

Published 5 December 2008

Online at stacks.iop.org/Nano/20/015302

\begin{abstract}
We report a new method for the fabrication of sub-10 nm nanopores in a fast single process step. The pore formation is accomplished by exploiting the competition between sputtering and deposition in ion-beam-induced deposition (IBID) on a thin membrane. The pore diameter can be controlled by adjusting the ion beam and gas exposure conditions. The pore diameter is well below the limit that can be achieved by focused ion beam (FIB) milling alone. There is no need of preparation and successive treatments. Apart from simplicity and speed, this method offers an additional advantage of a broad choice of material and thickness of the deposit and the membrane.
\end{abstract}

(Some figures in this article are in colour only in the electronic version)

\section{Introduction}

Nanopores have important applications in many fields of nanoscience: for example, for sensing single DNA molecules [1, 2], for localizing molecular-scale electrical junctions and switches [3, 4], as nanochannels in nanofluidic transistors [5], for fabricating point contacts [6, 7] and nanoelectrodes in electrochemistry [8], as aperture probes of near-field optical microscopy [9], as optical elements for subwavelength optical transmission devices [10], as nanostencil masks to create other nanostructures [11], and for measuring three-dimensional intensity profiles of laser focuses [12].

For simple pore fabrication, direct FIB milling of a thin membrane is commonly used. With a $5 \mathrm{~nm}$ full width at half maximum (FWHM) ion beam, Gierak et al fabricated sub$5 \mathrm{~nm}$ pores in a $20 \mathrm{~nm}$ ultra-thin $\mathrm{SiC}$ membrane [13]. With a signal feedback from an ion detector below the membrane, Patterson et al milled $18 \mathrm{~nm}$ diameter pores in a thick $\mathrm{Si}_{3} \mathrm{~N}_{4}$ membrane [14]. However, it is generally believed that fine-tuning of the pore size by FIB milling is difficult, and the resolution that can be achieved is limited by the beam diameter, beam shape and re-deposition, especially for thick membranes $[13,15]$. Pores in $\mathrm{Si}_{3} \mathrm{~N}_{4}$ or $\mathrm{SiO}_{2}$, which were initially fabricated by FIB milling or electron beam lithography, shrunk or grew upon exposure to a low-energy ion beam [16] or a high-energy electron beam [17]. The assumed mechanism is mass flow driven by surface tension. Feedback from an ion detector below the membrane [16] or visual feedback in a transmission electron microscope (TEM) [17] allows fine-tuning of the pore diameter with sub-nanometer resolution. Lo and Biance shrunk FIB milled pores in SiN by subsequent FIB scanning [18, 19]. Chang used a scanning electron microscope (SEM) to shrink pores in $\mathrm{Si}$ and $\mathrm{SiO}_{2}$, which were fabricated by micromachining [20]. Wu used laser heating to shrink mechanically punctured holes in thermal plastics [21]. Despite the extensive use, the mechanism of surface-tension-driven mass flow in pore shrinkage during broad-area beam scanning is still not fully understood [20, 22]. FIB milled pores can also be shrunk by thin film deposition on prefabricated pores, either by IBID [23-25], electron beam induced deposition (EBID) [26], low-pressure chemical vapor deposition (LPCVD) [15], or atomic layer deposition (ALD) $[27,28]$. However, the control of the final pore size is not straightforward. For instance, Schenkel et al observed that the larger of two initial pores became the smaller one during thin film deposition [23]. They related this effect to differences in the local supply of the precursor molecules.

Despite the large variety in pore fabrication techniques, it is still very desirable to develop simpler and faster methods. In this paper, we report a new method for the fabrication of sub-10 nm nanopores in a fast, single IBID step. The essence of our method is in controlling the dynamical balance between the sputtering and deposition involved in IBID. This can be achieved by adjusting the standard IBID parameters. In this work, we employed two very different precursor gases, one for metal $(\mathrm{Pt})$ deposition and the other one for insulator $\left(\mathrm{SiO}_{2}\right)$ deposition. 


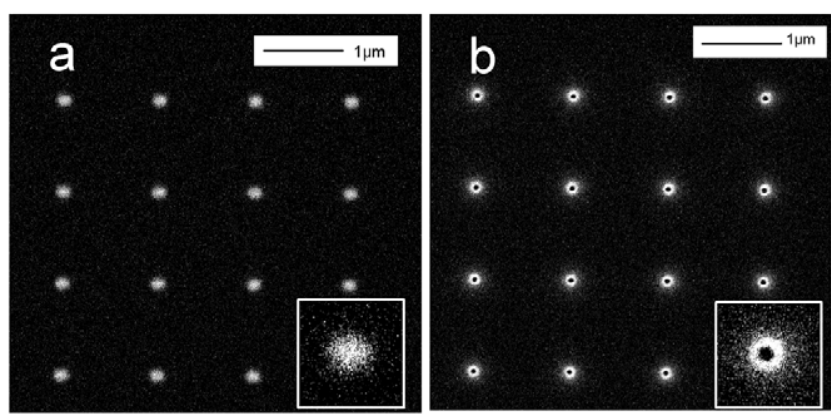

Figure 1. Ion beam current dependence of pore formation. SEM top views of Pt deposits on a $45 \mathrm{~nm}$ thick $\mathrm{Si}_{3} \mathrm{~N}_{4}$ membrane, fabricated (a) with $1 \mathrm{pA} 30 \mathrm{keV} \mathrm{Ga}^{+}$, only dots have formed; (b) with $8 \mathrm{pA}$ $30 \mathrm{keV} \mathrm{Ga}^{+}$, a pore has formed in the center of the dot. (The dwell time is $1 \mathrm{~ms}$ and the total exposure time is $1 \mathrm{~s}$ per dot.)

\section{Experimental details}

The precursor gas $\left(\mathrm{CH}_{3}\right)_{3} \mathrm{Pt}\left(\mathrm{C}_{\mathrm{P}} \mathrm{CH}_{3}\right)$ was the source for $\mathrm{Pt}$ deposition and TEOS that for $\mathrm{SiO}_{2}$ deposition. The IBID experiments were performed in two FEI dual beam systems, a STRATA DB235 for Pt deposition and a NOVA 600 for $\mathrm{SiO}_{2}$ deposition. The substrate was a double sided polished $\mathrm{Si}$ (100) wafer with $50 \mathrm{~nm}$ thick LPCVD $\mathrm{Si}_{3} \mathrm{~N}_{4}$ at both sides. The $\mathrm{Si}_{3} \mathrm{~N}_{4}$ membranes were fabricated by opening windows by dry etching in the nitride layer on the back side of the wafer and subsequent anisotropic etching of the $\mathrm{Si}$ wafer in $\mathrm{KOH}$ solution. The resulting $\mathrm{Si}_{3} \mathrm{~N}_{4}$ membranes were $400 \times 400 \mu \mathrm{m}^{2}$. The nitride layer after etching had a thickness of $45 \mathrm{~nm}$. A $30 \mathrm{keV}$ focused $\mathrm{Ga}^{+}$beam at normal incidence was used. The beam currents were 1,8 , and $13 \mathrm{pA}$. The FWHM of the incident beam is $10 \mathrm{~nm}(1 \mathrm{pA})$ and $12 \mathrm{~nm}(8$ and $13 \mathrm{pA})$. In the STRATA DB235, the chamber pressure was $2.3 \times 10^{-6} \mathrm{mbar}$ during IBID and the background pressure was $<1 \times 10^{-7} \mathrm{mbar}$. In the NOVA 600 , the pressures were $1.1 \times 10^{-5} \mathrm{mbar}$ and $4.3 \times 10^{-6} \mathrm{mbar}$, respectively. The nominal pumping speed is $2501 \mathrm{~s}^{-1}$ for nitrogen (as provided by FEI). Assuming that this value can be used for the precursor gas as well, the flow rate is thus about $1 \times 10^{-3} \mathrm{scc} \mathrm{s}^{-1}$. To ensure a constant precursor supply, the precursor was always introduced into the chamber at least $60 \mathrm{~s}$ before the ion beam. To reduce sample charging effects, first a few conductive Pt lines were deposited by IBID on the $\mathrm{Si}_{3} \mathrm{~N}_{4}$ membrane. They connect the insulating $\mathrm{Si}_{3} \mathrm{~N}_{4}$ membrane window to the bulk Si. Arrays of 16 pores were fabricated between these Pt lines. The ion beam was cycled continuously between these 16 spots. This procedure ensured sufficient precursor refreshment time, i.e. 15 times the beam dwell time on each single spot. The total exposure time equaled the dwell time multiplied by the number of executed cycles. On the same sample, a series of pore arrays was fabricated with either different dwell times or different exposure times. After fabrication of one or more series of arrays, the sample was transferred to a TEM for imaging. Imaging of the Pt deposits was performed in a field emission Philips CM200 TEM and of the $\mathrm{SiO}_{2}$ deposits in a FEI Tecnai (S)TEM. Both TEMs operated at an accelerating voltage of $200 \mathrm{kV}$. We studied the pore formation as a function of ion beam current, dwell time and exposure time.
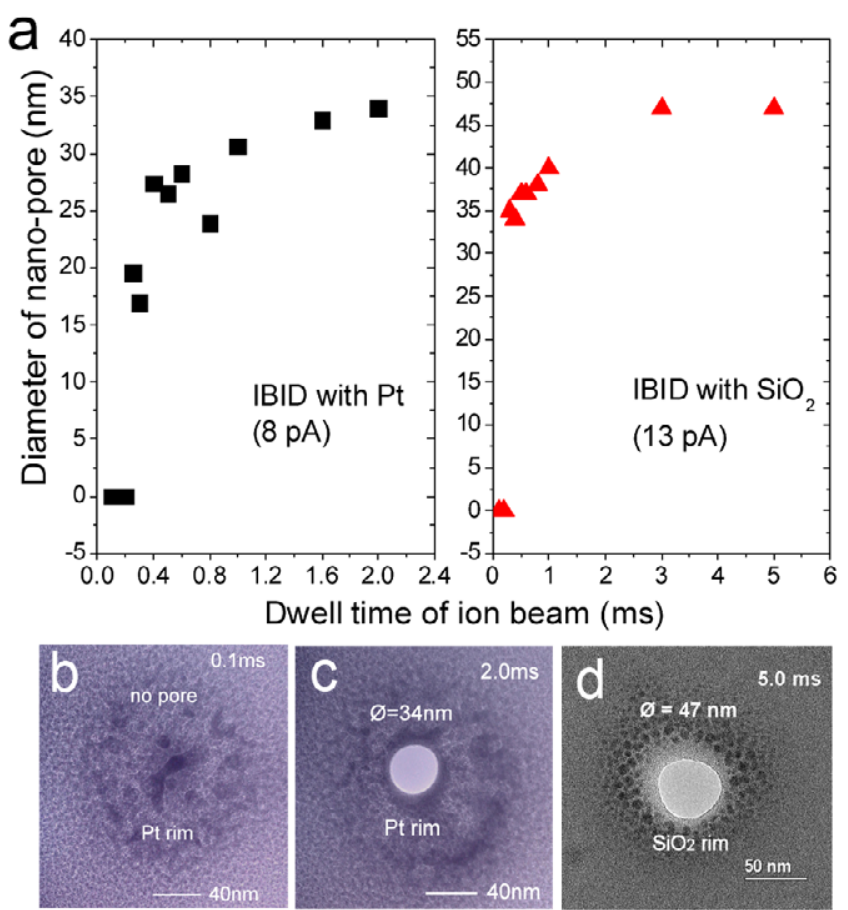

Figure 2. Dwell time dependence of pore formation. (a) Pore diameter versus dwell time for $\mathrm{Pt}$ or $\mathrm{SiO}_{2}$ depositions with 8 or $13 \mathrm{pA} 30 \mathrm{keV} \mathrm{Ga}^{+}$and exposure time of 4 or $2 \mathrm{~s}$ per pore, respectively. TEM top views of Pt deposit with dwell times of (b) $0.1 \mathrm{~ms}$ (no pores formed), (c) $2.0 \mathrm{~ms}$ (a central pore formed); (d) $\mathrm{SiO}_{2}$ deposit with a dwell time of $5.0 \mathrm{~ms}$.

\section{Results and discussion}

\subsection{Experimental results}

With a low current of $1 \mathrm{pA}$, only Pt dots were obtained (figure 1(a)). With $8 \mathrm{pA}$, pores formed in the center of the dots (figure 1(b)). With $24 \mathrm{pA}$, the pore diameter was considerably larger (not shown). In the results of figures $2-4$, an ion beam current of $8 \mathrm{pA}$ was chosen for Pt deposition and $13 \mathrm{pA}$ for $\mathrm{SiO}_{2}$ deposition.

To investigate the dwell time dependence of the pore diameter, a constant exposure time of $4 \mathrm{~s}$ per pore for $\mathrm{Pt}$ deposition and $2 \mathrm{~s}$ per pore for $\mathrm{SiO}_{2}$ deposition was chosen (figure 2). In both cases, the pore diameter increases with increasing dwell time. This trend decelerates for long dwell times. With short dwell times $(<0.3 \mathrm{~ms})$, no pores formed. With medium dwell times $(0.3-1.0 \mathrm{~ms})$, the pore diameter increases rapidly. And with long dwell times ( $>1.6 \mathrm{~ms}$ for $\mathrm{Pt}$ and $>3.0 \mathrm{~ms}$ for $\mathrm{SiO}_{2}$ deposition), the pore diameter stabilizes.

To investigate the exposure time dependence of the pore diameter, a medium dwell time of $0.5 \mathrm{~ms}$ was chosen for $\mathrm{Pt}$ deposition and $1.0 \mathrm{~ms}$ for $\mathrm{SiO}_{2}$ deposition (figure 3). At the early stage of exposure, the membrane was opened very rapidly ( $<0.5 \mathrm{~s}$ for $\mathrm{Pt}$ and $<1.0 \mathrm{~s}$ for $\mathrm{SiO}_{2}$ deposition). Then the pores closed slowly with increasing exposure time until they became fully closed (at $18 \mathrm{~s}$ for Pt deposition). The closing slows down with decreasing pore diameter. The smallest pores were $11 \mathrm{~nm}$ in diameter for $\mathrm{Pt}$ deposition and $5.5 \mathrm{~nm}$ for $\mathrm{SiO}_{2}$ deposition. For comparison, the diameter of several FIB milled pores is 

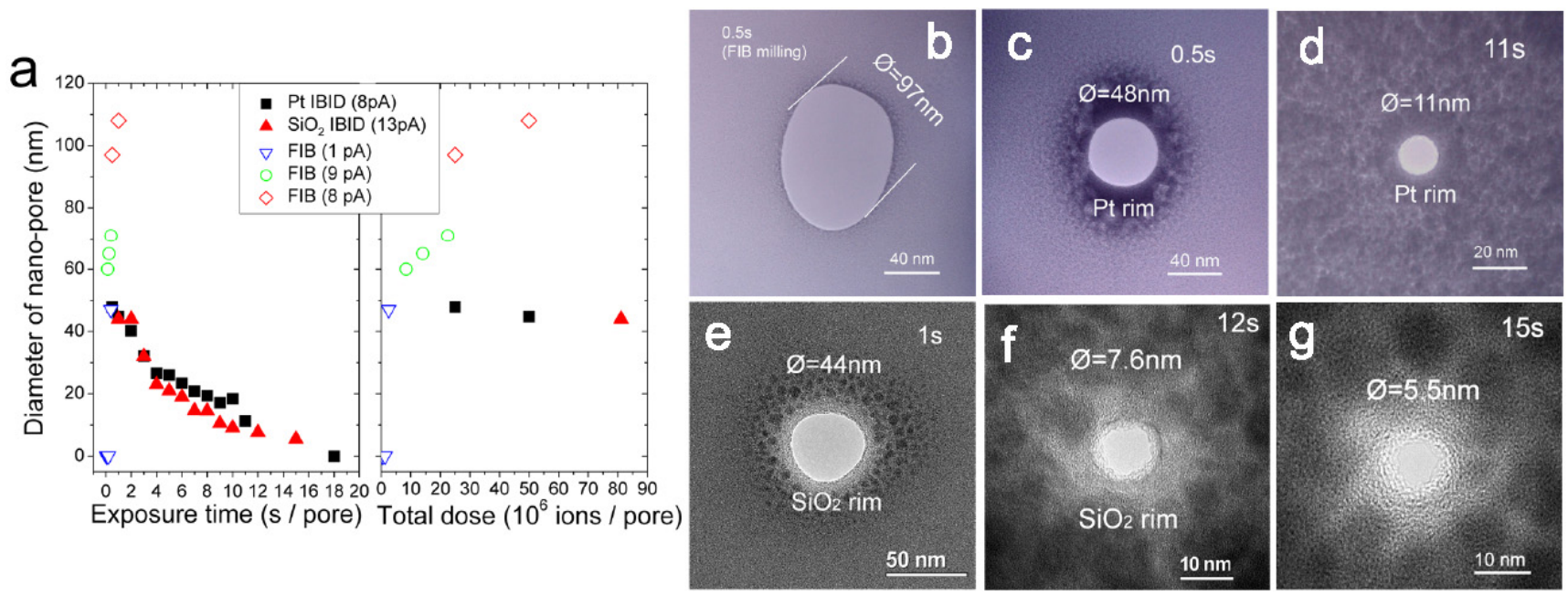

Figure 3. Exposure time dependence of pore formation. (a) Pore diameter versus exposure time. $\mathrm{Pt}$ or $\mathrm{SiO}_{2}$ depositions with 8 or $13 \mathrm{pA}$ $30 \mathrm{keV} \mathrm{Ga}^{+}$and dwell times of 0.5 or $1.0 \mathrm{~ms}$, respectively; TEM top views of (b) an FIB milled pore with an exposure time of $0.5 \mathrm{~s}$ per pore; pores fabricated by Pt deposition with exposure times of (c) $0.5 \mathrm{~s}$, (d) $11 \mathrm{~s}$ per pore; by $\mathrm{SiO}_{2}$ deposition with exposure times of (e) $1.0 \mathrm{~s}$, (f) $12 \mathrm{~s}$, and (g) $15 \mathrm{~s}$ per pore.
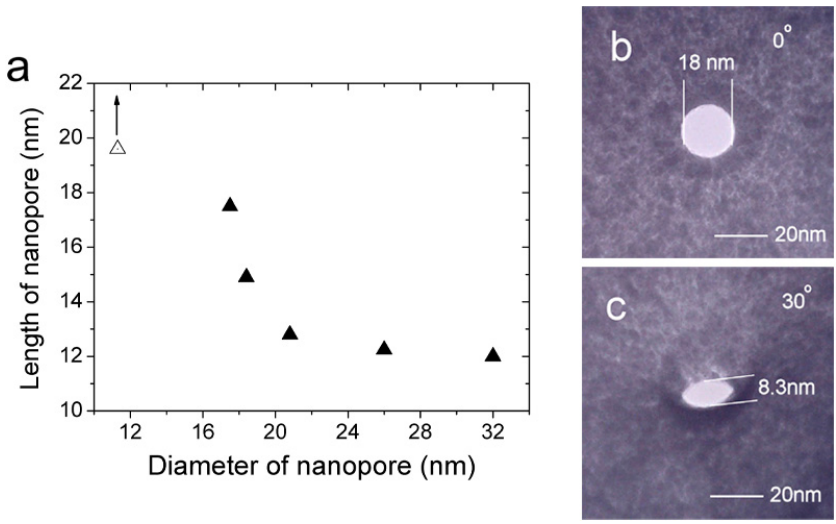

Figure 4. Pore shape. The pore is modeled as a cylinder in a sheet of material with constant thickness. That thickness is the pore length. (a) Measured pore length versus pore diameter. Pt deposits with $0.5 \mathrm{~ms}$ dwell time and various exposure times. The $11 \mathrm{~nm}$ pore must be at least $20 \mathrm{~nm}$ long since it was invisible in the $30^{\circ}$ tilted TEM image; TEM (b) top view of an $18 \mathrm{~nm}$ diameter pore; (c) tilted view of the pore in (b).

also shown in figure 3(a). Initially, they grew very rapidly. The growth continued until the pore diameter exceeded $100 \mathrm{~nm}$.

An investigation of the geometry of the nanopores is helpful both to understand the pore formation and to explore their possible applications. For simplicity we model our pore as a cylinder in a sheet of material with constant thickness. The pore length is the thickness of the sheet. By comparing $30^{\circ}$ tilted TEM views with top views, the pore length of the pores fabricated by Pt deposition was measured (figures 4(b) and (c)). We observed that the pore length increases with decreasing pore diameter. This trend accelerates when the pore diameter falls below $20 \mathrm{~nm}$ (figure 4(a)).

\subsection{Discussion}

We have observed that nanopores as small as $5.5 \mathrm{~nm}$ in diameter can be fabricated by a single-step IBID process. We found that the pore diameter can be controlled by adjusting the beam current, dwell time, or exposure time. It is well known that sputtering and deposition occur simultaneously in IBID. Sputtering is usually regarded as a negative factor that reduces the deposition rate and, therefore, most attention so far has been paid to suppress sputtering during IBID. This work shows that sputtering can also be useful in IBID. Exploration of new applications requires, however, a better understanding of the relation between sputtering and deposition. The mechanisms involved in IBID are deposition by (I) primary ions, (II) sputtered atoms, and (III) secondary electrons [29, 30]; see figure 5. Each mechanism has its own specific reaction zone around the impact site (figure 5(a)) [30]. The reaction zone of incoming primary ions (I) is comparable to the beam size. Secondary (sputtered) atoms leave the surface close to the impact site (II), while secondary electrons can travel a long distance in the solid before emission (III). In this work we exploited the competitions between sputtering and deposition in the various reaction zones to control the shape of the deposits. The control was achieved by adjusting either one of the following three standard IBID parameters: beam current, dwell time, or exposure time. Indeed, complex structures as donut-like deposits with a central tip (figure 5(b)) or with a central hole (figure 5(c)) have been obtained in a single IBID step at spot mode by adjusting the dwell time [30]. Below we propose a model that explains how these parameters affect the pore formation.

The sputtering yield $Y_{\mathrm{s}}$, expressed in number of sputtered atoms per incident ion, is independent of the ion beam density. On the other hand, the deposition yield $\mathrm{Y}_{\mathrm{d}}$, expressed in number of deposited atoms per incident ion, depends on the ratio between the ion beam density $j$ and the precursor flux $\phi$ [30-33]. For a fixed precursor flux, the deposition yield is independent of the ion beam density for low values of $j$, but decreases above a critical ratio between $j$ and $\phi$. Hence, for a low beam current at spot mode and a sufficient precursor flux, deposition dominates over sputtering and only dot-like deposits 

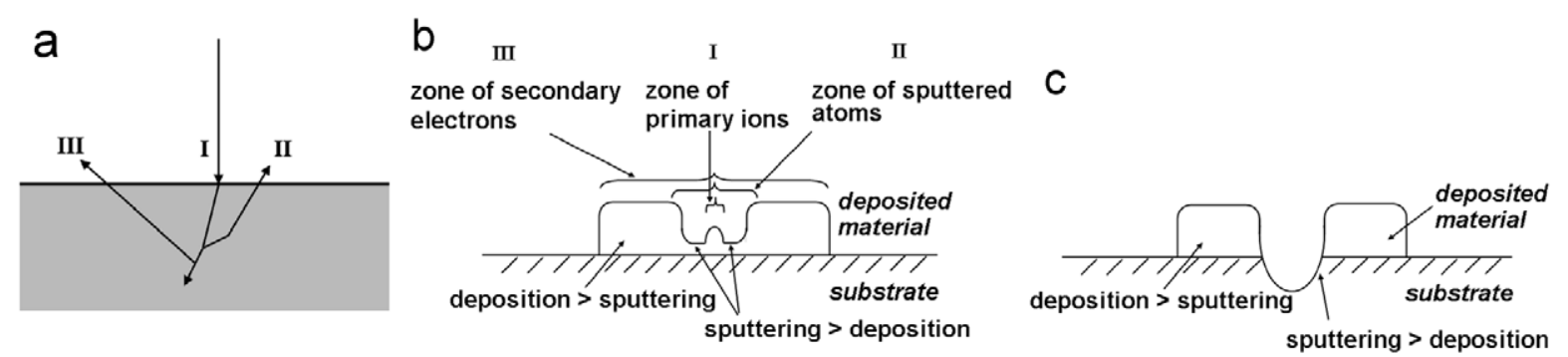

Figure 5. Balance between deposition and sputtering in IBID. Sketches of (a) activity regions of different particles contributing to IBID. I: incident primary ions, II: sputtered atoms, and III: secondary electrons. Reaction zones of I, II and III and donut-like structures with (b) a central tip, fabricated with a short dwell time, and (c) a central hole, with a long dwell time.

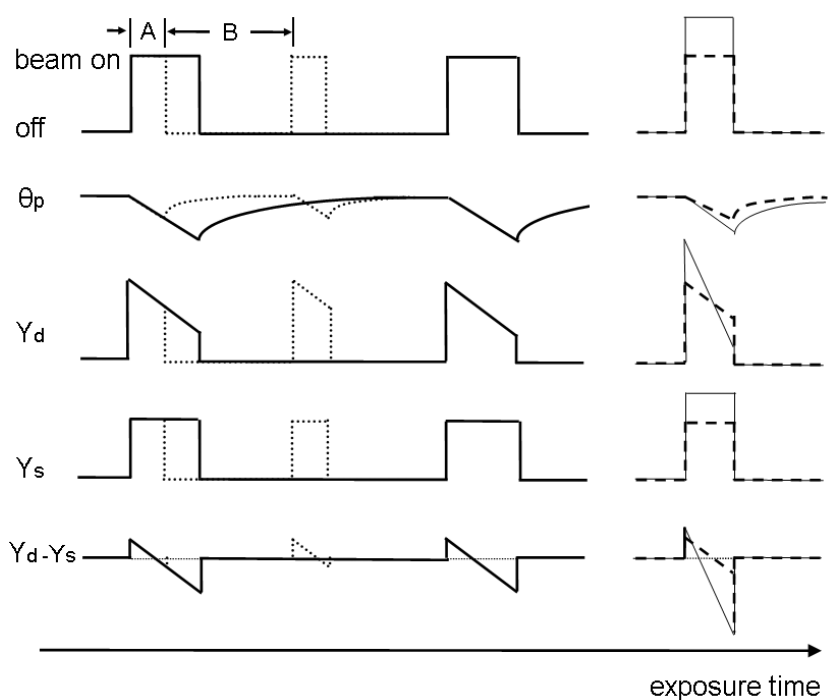

Figure 6. Sketch of the dwell time dependence of pore formation. Step A: beam-on. Step B: beam-off. $\theta_{\mathrm{p}}$ : precursor surface density. $Y_{\mathrm{d}}$ : deposition yield (which is proportional to $\theta_{\mathrm{p}}$ ). $Y_{\mathrm{s}}$ : sputtering yield (which is constant). $Y_{\mathrm{d}}-Y_{\mathrm{s}}$ : net deposition. Short dashed lines: short dwell times. Thick solid lines: long dwell times. The net deposition $Y_{\mathrm{d}}-Y_{\mathrm{s}}$, averaged over step A, is positive for short dwell times (short dashed lines) and negative for long dwell times (thick solid lines). At the right: medium dwell time. The beam current density affects the balance between deposition and sputtering: for a higher density (thin solid) the average net deposition is negative; for a lower density (long dashed) it is positive.

form (figure 1(a)). In contrast, for a sufficiently high beam current with the same gas flux and beam diameter, sputtering dominates in the center of the deposit, where the ion beam density is highest. Hence, a central pore forms (figure 1(b)). Still, deposition by secondary electrons and atoms [29, 30] dominates in the rim area, where sputtering by primary ions is low or absent.

With shorter dwell times, smaller pores form (figure 2). We discuss the dwell time and ion beam density dependencies of the pore formation via the sketch of figure 6 . In each cycle, adsorbed precursor molecules are being consumed at the beam spot during the beam-on step A. The adsorbed precursor layer is being refreshed during the beam-off step B. In our experiment the refreshment time is chosen to be long enough so that the precursor surface coverage $\theta_{\mathrm{p}}$ saturates again before the next cycle starts. The deposition yield $\mathrm{Y}_{\mathrm{d}}$

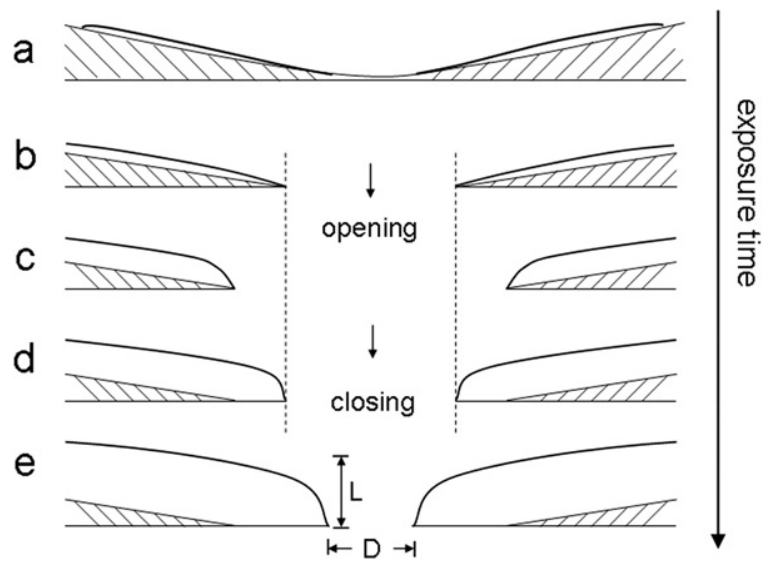

Figure 7. Sketch of the exposure time dependence of pore formation. (a)-(c): pores open; (c)-(e): pores close. $D$ and $L$ : pore diameter and length, respectively; white region: IBID deposits; shaded region: membrane.

strongly depends on the precursor surface coverage $\theta_{\mathrm{p}}$. In particular, it decreases during step A. Sputtering is caused by ion-solid interactions. Therefore, the sputtering yield $\mathrm{Y}_{\mathrm{s}}$ is independent of the precursor coverage; it remains constant during step A. For short dwell times (short dashed line in figure 6) the deposition yield exceeds the sputtering yield during most of the time of step A. Nevertheless, averaged over the entire step $A, Y_{d}-Y_{s}$ is positive and, thus, a deposit forms. On average, sputtering dominates over deposition for long dwell times (thick solid line). The balance between deposition and sputtering is delicate at medium dwell times. For the usual Gaussian-like beam profile, sputtering can dominate in the center of the beam spot (thin solid line), while deposition dominates in the rim (long dashed line). A small change in beam density or dwell time can flip the balance. Indeed, figure 2(a) shows that the pore diameter varies rapidly with dwell time just above a certain threshold.

For a dwell time just above the threshold, the membrane opens rapidly at the early stage of beam exposure (figure 3 ). The pores close again with increasing exposure time. We discuss the opening and closing process as sketched in figure 7. Initially (figure 7(a)) all ions interact with the membrane and therefore sputtering is strong. There is some deposition in the rim area. After the membrane has been opened, most ions pass through the pores without any interaction (figure 7(b)). 
However, the deposit in the rim continues to grow. When the thickness $L$ of the deposit increases, more precursor molecules adsorb on the pore's sidewall and more secondary electrons and atoms are emitted. Both effects enhance the deposition yield at the pore's sidewall. Therefore, the pore closes after figure 7(c). Note that the pore in figure 7(d) closes, although it has the same diameter $D$ as the pore in figure 7(b). We presume that deposition on the sidewall is mainly caused by secondary atoms and electrons; hence processes II and III in figure 5. The closing slows down with decreasing pore diameter until the pores are fully closed (figure 3(a)). Due to the high beam current density in the pore's center, the net deposition rate at the sidewall decreases with decreasing pore diameter. Moreover, the increasing aspect ratio of the pore (figure 4) might slow down the precursor supply to the pore's sidewall, thus slowing down the closing process even further. Actually, the slow rate of pore closing allows fine-tuning of the pore diameter.

The smallest pore diameter achieved so far is $5.5 \mathrm{~nm}$, which is less than 50\% of FWHM of the beam profile. Although it was possible to close a pore completely, it is not certain that at one moment the diameter was well below $5 \mathrm{~nm}$. Further investigations with preferably even smaller ion beams might reveal whether the biologically important diameters below $2 \mathrm{~nm}$ are achievable.

The IBID method for nanopore fabrication has several advantages over current techniques. It surpasses the main limitation for direct FIB milling, which is a rapid widening of the pore diameter caused by the ion intensity in the tail of the beam profile. A small variation in the membrane thickness [19] or a small instability in the beam current can result in a pore that is either too shallow or too wide. Contrary to the methods in which a wide pore is shrunk by successive processing steps [15-28], our method is based upon one single processing step. Techniques that are based on surface-tension-driven mass flow, such as ion beam sculpting [16] and electron beam induced drilling [17], have produced pores with diameters as small as $2 \mathrm{~nm}$. However, it is unclear whether these techniques can be applied to thick membranes and other materials as well. Our IBID method offers a broad choice of materials and thicknesses for both the membrane and the deposit.

In most FIB instruments there is a limited choice of beam currents. To fabricate small nanopores, the lowest current is preferred. The high sensitivity of the pore diameter for the dwell time (figure 2(a)) hinders the fabrication of very small pores by optimizing the dwell time. Probably, the best approach for the fabrication of pores with a required diameter is by selecting a dwell time just above the steep rise in figure 2 and optimizing the exposure time. A valuable option is a feedback system with an ion detector or a Faraday cup below the membrane - to stop the fabrication process when the required diameter is reached. Interestingly, the diameter of the smallest pores achieved in this work is less than half the ion beam diameter. The use of an even smaller beam [13] might make the fabrication of $1 \mathrm{~nm}$ pores feasible.

\section{Conclusions}

We have demonstrated a new method for the fabrication of nanopores through controlling the balance between sputtering and deposition during ion beam induced deposition (IBID). Sub-10 nm pores have been fabricated in a fast, single step. The pore diameter can be controlled by adjusting the standard IBID parameters.

The smallest pore obtained is $5.5 \mathrm{~nm}$ in diameter. This method has important advantages over the current nanopore fabrication techniques. It is fast-a few seconds per poreand only one processing step is required. Arrays of hundreds of pores can be made within reasonable time. Furthermore, the IBID pore fabrication method offers substantial freedom in the choices of membrane and deposit material and thickness. Pores in the sub-10 nm range exhibit a wide variety of potential applications, such as DNA sensors and point contacts. We foresee that further optimization of the IBID method will bring the important range below $2 \mathrm{~nm}$ within reach.

\section{Acknowledgments}

The authors like to thank V Kutchoukov (Delft University of Technology in the Netherlands) for $\mathrm{Si}_{3} \mathrm{~N}_{4}$ sample preparation and V J Gadgil (University of Twente in the Netherlands) for technical support. The authors also thank E van der Drift, V Sidorkin, K Hagen, P Kruit (all from Delft University of Technology) for their helpful discussions. The authors acknowledge the financial support by NanoNed.

\section{References}

[1] Dekker C 2007 Nat. Nanotechnol. 2209

[2] Rhee M and Burns M A 2007 Trends Biotechnol. 25174

[3] Zhou C, Deshpande M R and Reed M A 1997 Appl. Phys. Lett. 71611

[4] Ralph D C, Black C T and Tinkham M 1995 Phys. Rev. Lett. 743241

[5] Karnik R, Fan R, Yue M, Li D-Y, Yang P and Majumdar A 2005 Nano Lett. 5943

[6] Ralls K S, Buhrman R A and Tiberio R C 1989 Appl. Phys. Lett. $\mathbf{5 5} 2459$

[7] Wei H-X, Langford R M, Han X F and Coey J M D 2006 J. Appl. Phys. 99 08C501

[8] Krapt D, Wu M-Y, Smeets R M M, Zandbergen H W, Dekker C and Lemay S G 2005 Nano Lett. 6105

[9] Veerman J A, Otter A M, Kuipers L and Hulst N F V 1998 Appl. Phys. Lett. 723115

[10] Ghaemi H F, Thio T, Grupp D E, Ebbesen T W and Lezee H J 1998 Phys. Rev. B 586779

[11] Deshmukh M M, Ralph D C, Thomas M and Silcox J 1999 Appl. Phys. Lett. 751631

[12] Keyser U F, Krapt D, Koeleman B N, Smeets R M M, Dekker N H and Dekker C 2005 Nano Lett. 52253

[13] Gierak J et al 2007 Micro. Eng. 84779

[14] Patterson N, Adams D P, Hodges V C, Vasile M J, Michael J R and Kotula P G 2008 Nanotechnology 19235304

[15] Tong H D, Jansen H V, Gadgil V J, Bostan C G, Berenschot E, Rijn C J M and Elwenspoek M 2004 Nano Lett. 4283

[16] Li J, Stein, McMullan C, Branton D, Aziz M J and Golovchenko J A 2001 Nature $\mathbf{4 1 2} 166$

[17] Storm A J, Chen J H, Ling X S, Zandbergen H W and Dekker C 2003 Nat. Mater. 2537

[18] Lo C J, Aref T and Bezryadin A 2006 Nanotechnology 173264

[19] Biance A-L et al 2006 Micro. Eng. 831474

[20] Chang H, Iqbal S M, Stach E A, King A H, Zaluzec N J and Bashir R 2006 Appl. Phys. Lett. 88103109 
[21] Wu S S, Park S R and Ling X S 2006 Nano Lett. 62571

[22] Wu M-Y, Krapf D, Zandbergen H M and Batson P E 2005 Appl. Phys. Lett. 87113106

[23] Schenkel T, Radmilovic V, Stach E A, Park S J and Persaud A 2003 J. Vac. Sci. Technol. B 212720

[24] Allen F I, Persaud A, Park S J, Minor A, Sakurai M, Schneider D H and Schenkel T 2006 Nucl. Instrum. Methods B 244323

[25] Nilsson J, Lee J R I, Ratto T V and Létant S E 2006 Adv. Mater. 18427

[26] Danelon C, Santchi C, Brugger J and Vogel H 2006 Langmuir 2210711
[27] Chen P, Mitsui T, Farmer D B, Golovchenko J, Gordon R G and Branton D 2004 Nano Lett. 41333

[28] Chen P, Mitsui T, Farmer D B, Golovchenko J, Gordon R G and Branton D 2004 Nano Lett. 42293

[29] Shedd G M, Lezec H, Dubner A D and Melngailis J 1986 Appl. Phys. Lett. 491584

[30] Chen P, Alkemade P F A and Salemink H W M 2008 Japan. J. Appl. Phys. 475123

[31] Chiang T P, Sawin H H and Thompson C V 1997 J. Vac. Sci. Technol. A 153104

[32] Dubner A D and Wagner A J 1989 J. Vac. Sci. Technol. B 71950

[33] Dubner A D and Wagner A J 1989 J. Appl. Phys. B 73636 